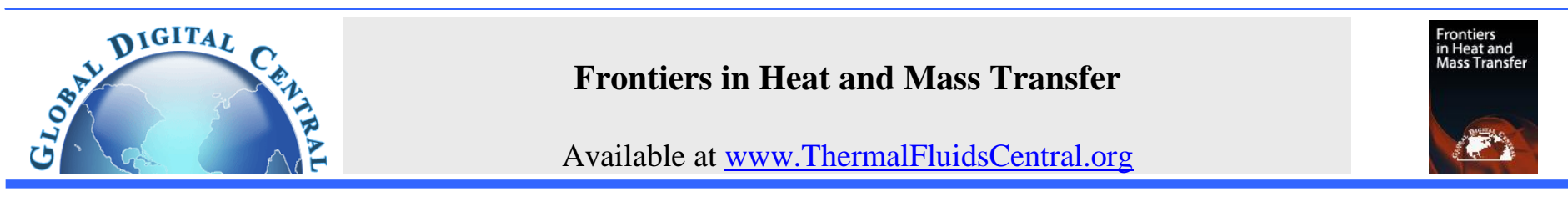

\title{
EFFECT OF THE VARIABILITY OF HEAT AND MASS TRANSFER COEFFICIENTS ON 3D UNSATURATED POROUS MEDIUM DRYING
}

\author{
Nidhal Ben Khedher ${ }^{\mathrm{a}, \mathrm{b}, *}$ \\ ${ }^{a}$ College of Engineering, Mechanical Engineering Department, Haïl University, Haïl City, 2440, Saudi Arabia \\ ${ }^{b}$ Laboratoire d'Études des Systèmes Thermiques et Énergétiques, Ecole Nationale d'Ingénieurs de Monastir, Monastir, 5019, Tunisie
}

\begin{abstract}
A Three-dimensional unstructured control volume finite method is developed to simulate unsteady coupled heat and mass transfer phenomena that arise during convective drying of unsaturated porous media. In order to simulate 3D geometries, as application here the drying of clay brick portion, we developed a Fortran code based on 3D unstructured meshes generated by the free mesh generator Gmsh. Several simulation results are presented and depict the effect of the variability of heat and mass transfer coefficients. These simulations prove that only three-dimensional model is able to capture the effect of variability of heat and mass transfer coefficients.
\end{abstract}

Keywords: Porous medium,3D drying, Gmsh, MVCEF, mass and heat transfer

\section{INTRODUCTION}

The high-energy consumption during porous solids drying has made it an interesting subject in a great number of industrial applications, including wood, food, textile, ceramics, building materials, etc (Caccavale et al., 2016; Almeida et al., 2013). Since, to reduce the drying time hence the energy consumption, we should simultaneously, improve both the circulation of water and gas-diffusion and accelerate surface evaporation. Under specific conditions, convective drying ensures not only the reduction of drying time but also preservation of the quality of the products at the same time. Therefore, heat and mass transfer should be carefully investigated throughout drying process.

However, the high cost and the time involved in experimental studies have made the numerical modeling widely used in analyzing the drying phenomena. Consequently, considerable researches have been conducted to numerically simulate the drying process (Defraeye, 2014; Lui et al., 2015; Sadrmomtazi and Haghi, 2008).

Drying is a process involves a coupled heat and mass transfer in a multi-phase flow in porous media. Based on the theory proposed by drying Luikov (Luikov, 1975) and later by Whitaker (Whitaker, 1977), several mathematical models for drying porous materials have been developed. The equations that govern the transport processes associated with flow in porous media are tightly coupled and highly non-linear. The discretization of these equations leads to a system of three partial differential equations for dynamic content variables as humidity, temperature and pressure. Several comprehensive theoretical twodimensional models are available for studying the drying process (Ben Khedher and Ben Nasrallah, 2010; Helel and Boukadida, 2008).

A review of the literature unveils little evidence of where unstructured mesh control volume method has been used to study numerically the heat and mass transfer in porous materials. Ferguson et al. (Ferguson and Turner, 1995a) have carried out a comparison of the finite element and control volume solution techniques applied to timber drying problems. Recently, structured mesh control volume techniques have been used to study the drying of wood (Ferguson and Turner, 1995b). Turner and Ferguson (Turner and Ferguson, 1995a) have used the finite element method to study similar problems. However, they found that the CVFEM was superior for reasons concerning efficiency, conservation, and robustness across a wide range of varying drying conditions of isotropic and anisotropic porous material. More recently, three-dimensional homogeneous wood drying models have been developed. In (Dedic et al., 2003), a simplified model based on the conservation of mass and energy using constant parameters obtained by comparing experimental data and numerical results for the drying of a block of beech wood was reported. Similarly, Perré and Turner (Perré and Turner, 1999), have developed a 3-D version of TransPore explaining a mass transfer model to simulate the drying of porous media. They showed that only three spatial dimensions are able to capture the correct physical behavior of the porous medium throughout the drying process and that the thickness and width of the board are necessary to account for the coupled mechanisms of thermal and mass diffusion, and Darcy's flow.

To date a very few of comprehensive theoretical three-dimensional models are available for studying the drying process. In previous works we developed a three dimensional tool for simulating the drying of a whole brick under industrial drying conditions (Rzig et al., 2017).

However, in the previous studies, the heat transfer coefficient was considered constant and the mass transfer coefficient was deduced by assuming analogy between heat and mass transfer. These two hypothesis lead to information deficiency as far as the real mechanism of heat and mass transfer during convective drying of porous media is concerned.

In this context, this study focuses on the effects of the variability of heat and mass transfer coefficients with surrounding air on the drying of clay brick. Consequently, the 3-D drying kinetics (average moisture content, temperature and pressure profiles) will be presented and analyzed.

\footnotetext{
*Corresponding Author Email: nidhal.ben.khedher@gmail.com
} 


\section{MATHEMATICAL METHOD}

Instead of simulating the drying of an entire brick (brick with 12 holes) and taking into account the symmetry of the problem, we will simulate heat and mass transfer in a single cavity. This square cavity is made of brick of length $1=30 \mathrm{~cm}$ and of width $\mathrm{L}=5.5 \mathrm{~cm}$. Only the internal cavity walls are exchanging heat and mass (Fig. 1)..

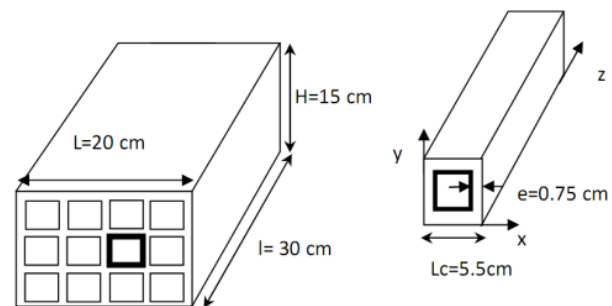

Fig. 1 porous brick cavity.

\subsection{Governing equations}

Based on Whitaker theory, a mathematical model governing heat and mass transfer is established for the unsaturated porous media.

In order to obtain a closed set of governing macroscopic equations, the following assumptions are made:

- $\quad$ The porous layer is homogenous and isotropic.

The solid, liquid and gas phases are in local thermodynamic equilibrium.

- $\quad$ The compression-work and viscous dissipation are negligible.

- $\quad$ The gas-phase is ideal in the thermodynamic sense.

diffusion term

The dispersion and tortuosity terms are interpreted as

The radiative heat transfer is negligible.

Considering these assumptions, macroscopic equations governing heat and mass transfer in the porous medium are:

\section{- Generalized Darcy's Law:}

The average velocities of $\left(\overline{V_{l}}\right)$ the liquid phase and the gas phase $(\overline{V g})$ are obtained using Darcy's Law which is generalized by using the concept of relative permeability defined as the ratio between the effective permeability and the intrinsic permeability.

- For the liquid phase:

$\overline{V_{l}}=-\frac{\mathrm{KK}_{\mathrm{l}}}{\mu_{\mathrm{l}}}\left[\nabla\left({\overline{\mathrm{P}_{\mathrm{g}}}}^{\mathrm{g}}-P_{c}\right)-{\overline{\rho_{l}}}^{\mathrm{g}}\right]$

With $P_{c}={\overline{P_{g}}}^{g}-\bar{P}_{l}^{l}$ : the capillary pressure.

- For the gas phase (without taking into consideration the gravitational effect):

$\overline{V_{g}}=-\frac{K K_{g}}{\mu_{g}} \nabla{\overline{P_{g}}}^{g}$

- Mass conservation equations:
- Liquid phase:

Assuming that liquid density is constant, the mass conservation equation of the liquid phase is:

$\frac{\partial \varepsilon_{l}}{\partial t}+\nabla\left(\overline{\mathrm{V}_{1}}\right)=-\frac{\dot{m}}{\bar{\rho}_{1}}$

Where $\tilde{m}$ is the mass rate of evaporation and $\varepsilon_{\mathbb{l}}$ is the volume fraction of liquid phase.

- Gas phase:

For this phase the average density is not constant. In this case, the mass conservation equation of the gas phase is given by:

$\frac{d \overline{\rho_{g}}}{d t}+\nabla\left(\bar{\rho}_{g}^{g} \overline{V_{g}}\right)=\dot{m}_{g}$

Where ${ }^{-\frac{-g}{g}}$ is the intrinsic average density of the gas phase. This phase is considered as an ideal mixture of perfect gases.

- Vapor phase:

$\frac{d \overline{\rho_{v}}}{d t}+\nabla\left(\overline{\rho_{v}^{g}} \overline{V_{v}}\right)=\dot{m}_{v}$

$\bar{\rho}_{v}^{g} \bar{V} v=\bar{\rho}_{v}^{g} \bar{V} g-\rho_{\mathrm{g}}^{\mathrm{g}} \mathrm{D}_{\mathrm{eff}} \nabla\left(\frac{\overline{\rho_{\mathrm{v}}}}{\overline{\rho_{\mathrm{g}}}}\right)$

$\mathrm{D}_{\text {eff }}$ represents the coefficient of the effective diffusion of the vapor in the porous medium. This coefficient takes into account the resistance to the diffusion due to tortuosity and the effects of constriction.

- Energy conservation equation:

$\frac{\partial}{\partial t}\left(\overline{\rho \mathrm{C}_{\mathrm{p}}} \overline{\mathrm{T}}\right)+\operatorname{div}\left[\left({\overline{\rho_{1}}}^{\mathrm{l}} \mathrm{C}_{\mathrm{pl}} \overline{\mathrm{v}_{1}}+\sum_{\mathrm{k}=\mathrm{a}, \mathrm{v}}{\overline{\rho_{\mathrm{k}}}}_{\mathrm{g}}^{\mathrm{g}} \mathrm{pk}_{\mathrm{pk}} \overline{\mathrm{v}_{\mathrm{k}}}\right) \bar{T}\right]$

$=\nabla\left(\lambda_{\text {eff }} \cdot \nabla \overline{\mathrm{T}}\right)-\Delta H_{\text {vap }} \cdot \dot{\mathrm{m}}_{v}$

$\Delta H_{\mathrm{vap}}$ is the latent heat of vaporization at temperature $\mathrm{T}(\mathrm{K})$.

$\lambda_{\text {eff and }} \overline{\rho C_{p}}$ are respectively the effective thermal conductivity and the constant pressure heat capacity of the porous medium and $\overline{\rho C_{p}}$ is given by:

$\overline{\rho C_{p}}=\bar{\rho}_{s} C_{p s}+\bar{\rho}_{l} C_{p l}+\bar{\rho}_{a} C_{p a}+\bar{\rho}_{v} C_{p v}$

Where ${ }^{\rho_{\mathrm{s}}} \mathrm{C}_{\mathrm{ps}}, \bar{\rho}_{\mathrm{l}} \mathrm{C} \mathrm{pl}, \bar{\rho}_{\mathrm{v}} \mathrm{C}_{\mathrm{pv}}$ and ${ }^{-} \bar{\rho}_{\mathrm{a}} \mathrm{C}_{\mathrm{pa}}$ are respectively the mass heat capacities of the brick solid matrix, liquid, vapor and air.

- Thermodynamic relations:

The partial pressure of the vapor is equal to its equilibrium pressure:

$P_{v}=P_{v e q}(T, S)$ 
Where $\mathrm{S}$ is the liquid saturation defined by:

$$
S=\frac{\varepsilon_{l}}{\varepsilon}
$$

The gaseous phase is assumed to be an ideal mixture of perfect gases:

$$
\begin{aligned}
& \overline{\mathrm{P}}_{\mathrm{i}}=\frac{\bar{\rho}_{\mathrm{i}}}{\mathrm{M}_{\mathrm{i}}} \mathrm{RT} ; \mathrm{i}=\mathrm{a}, \mathrm{v} \\
& \overline{\mathrm{P}}_{\mathrm{g}}=\overline{\mathrm{P}}_{\mathrm{a}}+\overline{\mathrm{P}}_{\mathrm{v}}, \bar{\rho}_{\mathrm{g}}=\bar{\rho}_{\mathrm{a}}+\bar{\rho}_{\mathrm{V}}
\end{aligned}
$$

$$
\frac{P v}{P v s}=\exp \left(-\frac{2 \cdot \sigma \cdot \mathrm{M}_{v}}{r \cdot \rho_{l} \cdot R \cdot T}\right)
$$

\subsection{Boundary conditions}

Initially, the temperature, the gas pressure and liquid saturation are uniform in the brick.

Thermal energy brought by air convection is necessary for water evaporation and to the heat conduction in porous medium. This energy is function of temperature and heat transfer coefficient (Fig. 3).

On the exchanging faces (internal faces of the cavity), we can write:

$$
\left[\lambda_{\text {eff }} \frac{\partial\langle T\rangle}{\partial X_{i}}+\Delta H_{v a p} \rho_{l}\left\langle V_{l}\right\rangle n_{i}\right]=h_{t x}\left(\langle T\rangle-T_{\infty}\right)
$$

The mass flow corresponding to evaporation and to the evacuation of water is function of the vapor density difference and mass transfer coefficient:

$\left[\rho_{l}\left\langle V_{l}\right\rangle+\left\langle\rho_{v}\right\rangle^{g}\left\langle V_{v}\right\rangle\right] n_{i}=h_{m x}\left(C_{v s}-C_{v \infty}\right)$

The gas pressure on exchanging face is equal to atmospheric pressure:

$$
\left[\left\langle P_{g}\right\rangle^{g}\right]=P_{\text {atm }}
$$

Because of the symmetry of the problem the external faces of the cavity are kept impermeable.

$$
\begin{aligned}
& {\left[\lambda_{\text {eff }} \frac{\partial\langle T\rangle}{\partial X_{i}}+\Delta H_{v a p} \rho_{l}\left\langle V_{l}\right\rangle n_{i}\right]=0} \\
& {\left[\rho_{l}\left\langle V_{l}\right\rangle+\left\langle\rho_{v}\right\rangle^{g}\left\langle V_{v}\right\rangle\right] n_{i}=0} \\
& {\left[\frac{\partial\left\langle P_{g}\right\rangle^{g}}{\partial X_{i}}\right]=0}
\end{aligned}
$$

In this study, we will test the variability of heat and mass transfer coefficients on the drying phenomena. The convective heat and mass transfer coefficients were listed in Table 1:

For planar brick faces (Incropera et al., 2002), the local coefficients of heat transfer $\left(\mathrm{h}_{\mathrm{tx}}\right)$ and mass transfer $\left(\mathrm{h}_{\mathrm{mx}}\right)$ are function of ambient fluid parameters (temperature, vapor concentration, pressure, velocity).
Table 1Convective heat and mass transfer coefficients.

\begin{tabular}{|c|c|}
\hline$h_{t}$ & $\frac{\lambda \times 0.023 \times R_{e}^{4 / 5} \times P_{r}^{1 / 3}}{L_{c}}$ \\
\hline$h_{m}$ & $\frac{D_{A, B} \times 0.023 \times R_{e}^{4 / 5} \times S_{c}^{1 / 3}}{L_{c}}$ \\
\hline Validity & $R_{e}<5 \times 10^{5}$ \\
& $P_{r} \geq 0.6$ \\
& $S_{c} \geq 0.6$ \\
\hline$R_{e}$ & $\frac{\rho_{a} w_{a} L_{c}}{\mu_{a}}$ \\
\hline$P_{r}=\frac{C_{a} \mu_{a}}{\lambda_{a}} \quad S_{c}=\frac{\vartheta_{a}}{D_{A, B}}$ \\
\hline
\end{tabular}

With:

$h_{t}:$ the convective heat transfer coefficient $\left(\mathrm{W} / \mathrm{m}^{2}{ }^{\circ} \mathrm{C}\right)$

$h_{m}:$ the convective mass transfer coefficient $(\mathrm{m} / \mathrm{s})$

$D_{A, B}$ is the vapor diffusion in air given by:

$D_{A, B}=D_{\text {vap, air }}=0.26 \times 10^{-4}\left(\mathrm{~m}^{2} / \mathrm{s}\right)$

$L_{c}$ is the characteristic length of brick.

\section{SOLUTION METHOD}

The equations set, with initial and boundary conditions has been solved numerically using the Control Volume Finite Element Method (CVFEM). The advantages of this method are (i) It ensures the flux conservation (ii) the used control volumes present more faces, which makes it possible to avoid the numerical diffusion. (iii) The control volume is composed of triangular elements that improve the grid flexibility (Figure2). For additional details the reader can refer to (Rzig et al., 2017).

\subsection{Mesh Generation}

For the mesh generation (Fig. 2), the free mesh generator Gmsh was employed (Geuzaine and Remacle, 2009). The brick domain is divided first in six-node prisms. To create the polygonal control volumes around each node in the finite element grid, the centroids of the triangular elements (bases of prisms) are joined to the midpoints of the corresponding sides (Fig. 2). Then we developed a Fortran program called "Geomet Generator" allowing the reading of the Gmsh mesh file (*.msh) and the construction of control volumes. 


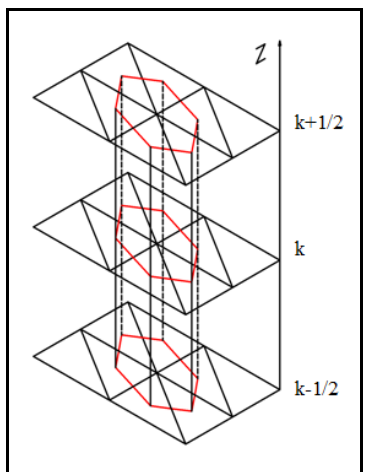

Fig. 2 Control volume and sub-volume.

Geomet Generator generates a 'Geomet.g' file containing the control volumes Data structures. This file will be then used by our main program called "porous drying simulator". In previous works the code was validated and for further details reader can refer to (Rzig et al., 2017).

The grid dependency is checked as to obtain the suitable mesh size for which the drying time does not depend upon the mesh size or is independent of the number of nodes. The effect of grid dependency on drying time is shown in Table 2.

Table 2 Grid-independency examination.

\begin{tabular}{|l|l|}
\hline Number of nodes & Drying time \\
\hline 2900 & $17 \mathrm{~h}$ \\
\hline 8700 & $15 \mathrm{~h} 50 \mathrm{~min}$ \\
\hline 14500 & $15 \mathrm{~h} 46 \mathrm{~min}$ \\
\hline
\end{tabular}

As seen in table 2, there were almost no differences in the numerical solutions in high number meshed cases over 8700 nodes. Therefore, the computational domain with 8700 nodes was used for all numerical simulations.

\section{RESULTS AND DISCUSSIONS}

This section is devoted to the numerical results obtained from the compilation of numerical model which is applied to the drying of porous brick.

To numerically simulate the three-dimensional phenomenon of drying of the portion of the brick, we use free mesh generator Gmsh to achieve three-dimensional meshing (Fig. 3).

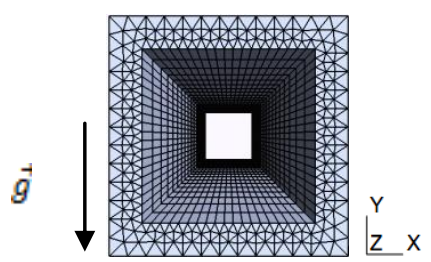

Fig. 3 Mesh generation by Gmsh.

The operating conditions are taken the same of a typical industrial drying process which are listed in Table 3.
Table 3Operating conditions.

\begin{tabular}{|c|c|c|c|c|c|}
\hline $\begin{array}{c}\mathrm{T}_{\mathrm{amb}} \\
\left({ }^{\circ} \mathrm{C}\right)\end{array}$ & $\begin{array}{c}\mathrm{T}_{\text {ini }} \\
\left({ }^{\circ} \mathrm{C}\right)\end{array}$ & $\begin{array}{c}\mathrm{S}_{\text {ini }} \\
(\%)\end{array}$ & $\mathrm{C}_{\mathrm{vamb}}$ & $\begin{array}{c}\mathrm{P}_{\mathrm{amb}} \\
(\mathrm{atm})\end{array}$ & $\begin{array}{c}\mathrm{P}_{\text {ini }} \\
(\mathrm{atm})\end{array}$ \\
\hline 100 & 20 & 50 & 0 & 1 & 1 \\
\hline
\end{tabular}

From the time evolution of temperature, liquid saturation and gaseous pressure for four nodes aligned along the $\mathrm{z}$ axis (Figs. 4-6) where local coefficients of heat transfer (htx) and mass transfer (hmx) are employed, we can clearly observe the three conventional drying phases:

- $\quad$ The transient heating phase

- The constant drying rate phase

- $\quad$ The decreasing drying rate phase:

- $\quad$ First decreasing drying rate period

- $\quad$ Second decreasing drying rate period

However, from Fig. 4, it is noted that the isenthalpic phase is longer for the nodes away from the hot air inlet section. This is mainly due to the variation of transfer coefficients which have high values in the vicinity of the inlet $(\mathrm{z}=0)$ whereas they decrease rapidly away from this zone. Far from the input section, where the coefficients are much lower, a very short isenthalpic phase is observed.

A preliminary analysis of the slab temperature, gas pressure and water saturation profiles (Figs. 4-6) shows that the variability of heat and mass transfer coefficients with z-direction has an appreciable effect on these state variables.

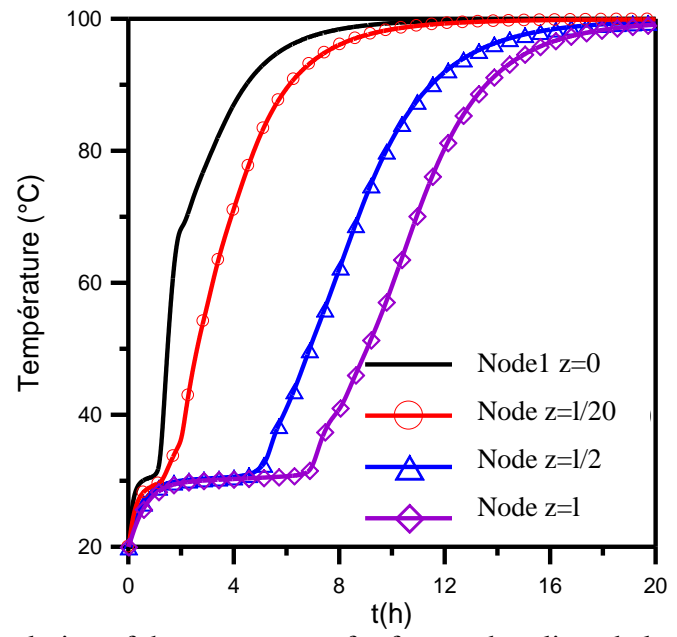

Fig. 4 Evolution of the temperature for four nodes aligned along the $\mathrm{z}$ axis.

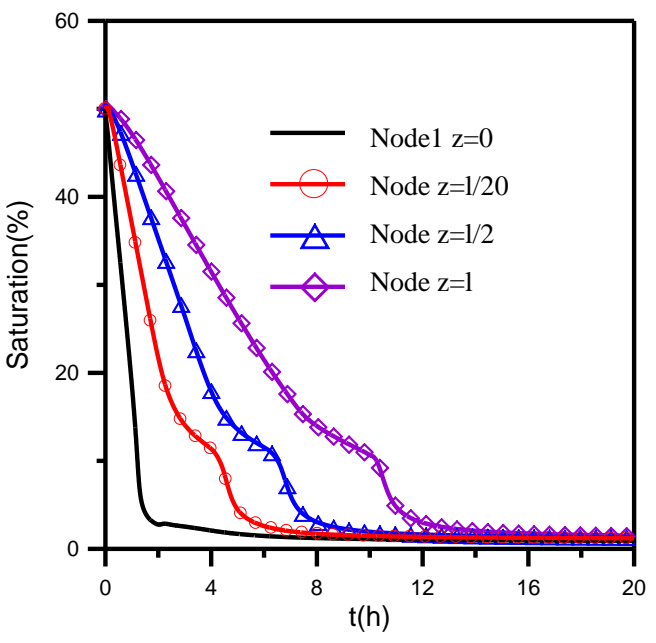

Fig. 5 Evolution of the liquid saturation for four nodes aligned along the $\mathrm{z}$ axis. 


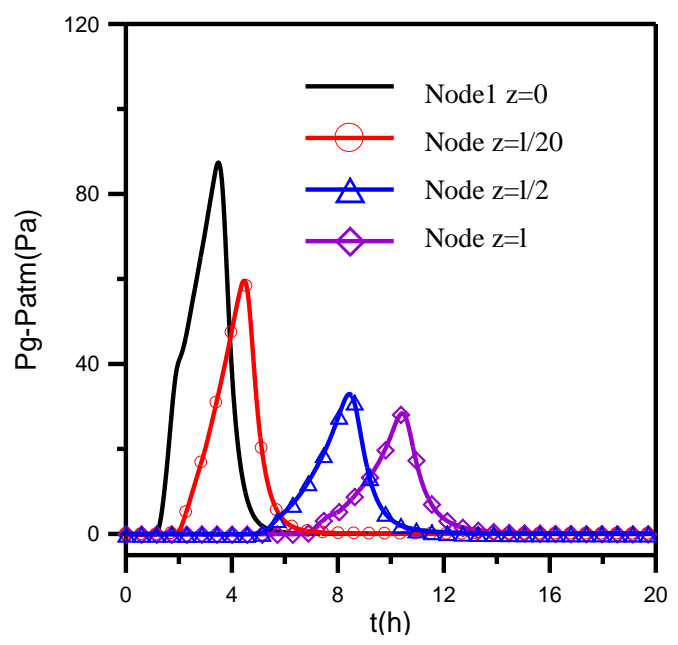

Fig. 6 Evolution of the gaseous pressure for four nodes aligned along the $\mathrm{z}$ axis.

In order to better observe the effect of the variability of the coefficients, we have shown in Figs. 7-8 the three-dimensional distribution of the temperature, the liquid saturation and the pressure in water in the medium at different times.

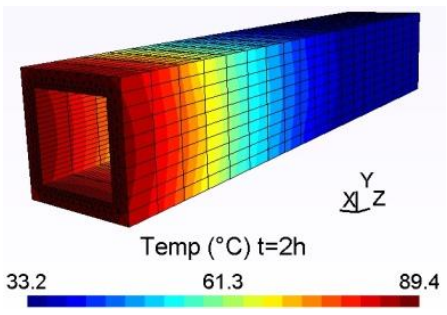

(a)

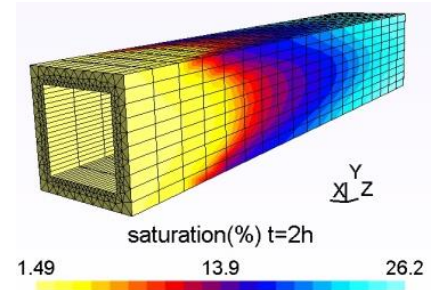

(b)

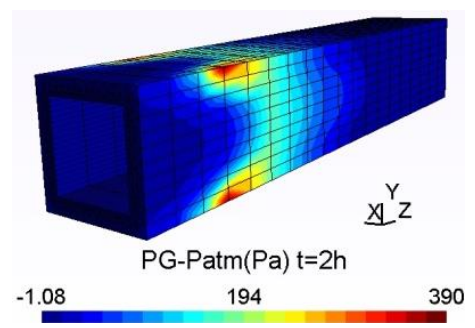

(c)

Fig. 7 Three-dimensional distribution of: (a) temperature, (b) liquid saturation and (c) gaseous pressure during the convective drying of brick portion after $2 \mathrm{~h}$

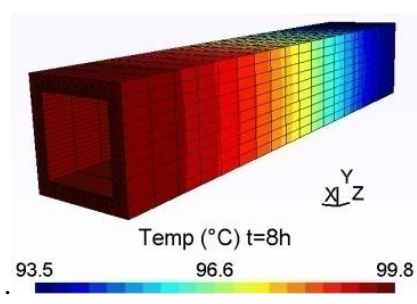

(a)

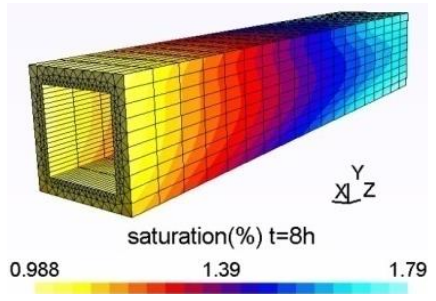

(b)

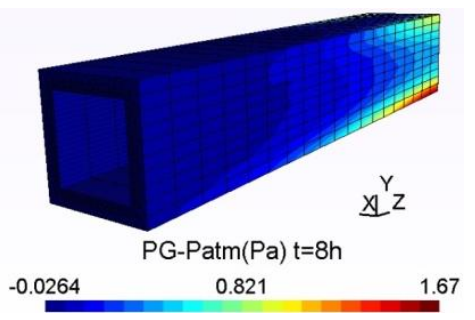

(c)

Fig. 8 Three-dimensional distribution of temperature, liquid saturation and gaseous pressure during the convective drying of brick portion after 8 h.

Figure 7 shows the displacement of the drying front dividing the porous medium into two regions: one wet and where the temperature is near the wet temperature and the other dry where the temperature increases significantly. The drying front appears earlier in the vicinity of the inlet section. This is due to the intensity of the transfers in the vicinity of the input. This figure shows that the mass and thermal transfers are not two-dimensional.

After $8 \mathrm{~h}$ of drying, Fig. 8 shows the non-symmetry of the saturation field along the y-axis under the effect of gravity. This behavior can be explained by the gravity effect since water migrates by gravity to the bottom of the brick portion(Fig. 8 (b)).

\section{CONCLUSION}

The present work constitutes a contribution to the understanding of the phenomena involved in heat and mass transfer mechanisms during the drying process in a porous medium. A three-dimensional heat and mass transfer model based on Whitaker's theory has been established. We simulated the case of drying by entrainment, a porous medium (brick), dried by an air flow.

For the discretization of the equations, we have adopted the method of control volumes based on finite elements. The effect of the heat and mass transfer coefficients has been studied and it has been shown that it is necessary to take into account the effect of the dynamic and mass thermal boundary layers on the heat and mass transfer coefficients. Consequently, we can conclude that not considering the variability of heat and mass transfer coefficients could cause significant errors. 


\section{NOMENCLATURE}

$C_{a} \quad$ specific heat of the air $(\mathrm{kJ} / \mathrm{kgK})$

$C_{p} \quad$ specific heat at constant pressure $(\mathrm{kJ} / \mathrm{kgK})$

$C_{v} \quad$ specific heat of the vapor $(\mathrm{kJ} / \mathrm{kgK})$

$C_{w} \quad$ specific heat of the water $(\mathrm{kJ} / \mathrm{kgK})$

$D_{A, B}$ diffusion coefficient $\left(\mathrm{m}^{2} / \mathrm{s}\right)$

D holes diameter (m)

g gravitational acceleration $\left(\mathrm{m} / \mathrm{s}^{2}\right)$

$h_{m} \quad$ convective mass transfer coefficient $(\mathrm{m} / \mathrm{s})$

$h_{t} \quad$ convective heat transfer coefficient $\left(W / \mathrm{m}^{2} \mathrm{c} \mathrm{C}\right)$

$K \quad$ intrinsic permeability $\left(\mathrm{m}^{2}\right)$

$L_{c} \quad$ characteristic length of brick (m)

$M_{a} \quad$ molar mass of air $(\mathrm{kg} / \mathrm{mol})$

$M_{v} \quad$ molar mass of vapour $(\mathrm{kg} / \mathrm{mol})$

$\dot{\mathrm{m}}$ evaporation rate $(\mathrm{kg} / \mathrm{s})$

$n_{\mathfrak{i}} \quad$ outward normal vector

$P \quad$ pressure $(\mathrm{Pa})$

$P c \quad$ capillary pressure $(\mathrm{Pa})$

$R_{V g} \quad$ partial pressure of saturated vapour $(\mathrm{Pa})$

$R \quad$ gas constant $\mathrm{kJ} / \mathrm{kmol} . \mathrm{K}$

$r \quad$ characteristic magnitude that represents the average radius of curvature of the menisci if the retention forces of the liquid are of capillary origin

$S \quad$ liquid saturation (\%)

$T$ temperature (K)

$t$ time (s)

$w_{a} \quad$ air velocity $(m / s)$

\section{Greek symbols}

$\varepsilon \quad$ porosity

घ] volume fraction of liquid phase

$\mu \quad$ dynamic viscosity $(\mathrm{kg} / \mathrm{ms})$

$\vartheta \quad$ kinematic viscosity $\left(\mathrm{m}^{2} / \mathrm{s}\right)$

\section{Subscripts}

O initial condition

a air

eff effective

$x \quad$ local

\section{REFERENCES}

Almeida, G.S., Da Silva, J.B., E Silva, C.J., Swamakar, R., Neves, G.A., and De Lima, A.G.B., 2013, "Heat and Mass Transport in an Industrial Tunnel Dryer: Modeling and Simulation Applied to Hollow Bricks," Applied Thermal Engineering, 55, 78-86. https://doi.org/10.1016/j.applthermaleng.2013.02.042

Ben Khedher, N., and Ben Nasrallah, S., 2010, "Unstructured Control Volume Finite Element Method for Coupled Heat and Mass Transfer during the Drying of Porous Medium having Complex 2D-Geometry," International Journal of Heat and Technology, 28(2), 133-139. https://doi.org/10.18280/ijht.280211

Caccavale, P., De Bonis, M.V., and Ruoccob, G., 2016, "Conjugate heat and mass transfer in drying: A modeling review," Journal of Food Engineering, 176, 28-35.

https://doi.org/10.1016/j.jfoodeng.2015.08.031.

Dedic, A.D., Mujumdar, A.S., and Voronjec, D.K., 2003, “A Three Dimensional Model for Heat and Mass transfer in Convective Wood Drying," Drying Technology, 21(1), 1-15. https://doi.org/10.1081/DRT-120017280

Defraeye, T., 2014, "Advanced computational modelling for drying processes - A review," Applied Energy, 131, 323-344.

https://doi.org/10.1016/j.apenergy.2014.06.027

Ferguson, W., and Turner, I.W., 1995a, "A Comparison of the Finite Element and Control Volume Solution Techniques Applied to Timber Drying Problems Below the Boiling Point," Int. J. Num. Meth. Eng., 38(9), 451-467.

https://doi.org/10.1002/nme.1620380307

Ferguson, W., and Turner, I.W., 1995b, “A Study of Two-Dimensional Cell-Centered and Vertex-Centered Control-Volume Schemes Applied to High Temperature Timber Drying," J. Num. Heat Transfer, Part B: Fundamentals, 27, 393-415.

https://doi.org/10.1080/10407799508914964

Geuzaine, C., and Remacle, J.F., 2009, "Gmsh: A 3-D Finite Element Mesh Generator with Built-in Pre- and Post-Processing Facilities," International Journal for Numerical Methods in Engineering, 79(11), 1309-1331.

https://doi.org/10.1002/nme.2579

Helel, D.E., and Boukadida, N., 2008, "Heat and mass transfer during drying process," International Journal of Heat and Technology, 26(2), 3-25.

https://doi.org/10.18280/ijht.260204

Incropera, F.P., DeWitt, D.P., Bergman, T.L., and Lavine, A.S., 2002, Fundamentals of Heat and Mass Transfer, John Wiley \& Sons, New York, USA.

Liu, L., Sun, Z., Wan, C., and Wu, J., 2015, “Jet Flow Field Calculation \& Mechanism Analysis On Hot-Air Drying Oven Based On Rng K-E Model," International Journal of Heat and Technology, 33, 77-82. https://doi.org/10.18280/ijht.330111

Luikov, A.V., 1975, "Systems of Differential Equations of Heat and Mass Transfer in Capillary Porous Bodies (Review)," Int. J. Heat Mass Transfer, 18, 1-14.

Perré, P., and Turner, I.W., 1999, “A 3-D Version of TransPore: a Comprehensive Heat and Mass Transfer Computational Model for Simulating the Drying of Porous Media," Int. J. Heat Mass Transfer, 42, 4501-4521. https://doi.org/10.1016/S0017-9310(99)00098-8

Rzig, R., Ben Khedher, N., Ben Nasrallah, S., 2017, "Threedimensional simulation of mass and heat transfer during unsaturated porous medium drying," Heat Transfer Research, 48(11), 985-1005 
https://doi.org/10.1615/HeatTransRes.2017016243.

Sadrmomtazi, A., and Haghi, A.K., 2008, "A numerical study on thermal drying of moist brick," International Journal of Heat and Technology, 26(1), 61-65. https://doi.org/10.18280/ijht.260109.

Turner, I.W., and Ferguson, W.J., 1995a, "An Unstructured Mesh CellCentered Control Volume Method for Simulating Heat and Mass
Transfer in Porous Media: Application to Softwood Drying," Part I: The Isotropic Model. Appl. Math. Modeling, 19(11), 654-667. https://doi.org/10.1016/0307-904X(95)00087-Z

Whitaker, S., 1977, Simulation Heat, Mass and Momentum Transfer in Porous Media a Theory of Drying. Advances in Heat Transfer, Academic Press, New York, USA 